\title{
REFERENCE INSTRUMENTS BASED ON SPECTROMETRIC MEASUREMENT WITH LUCAS CELLS
}

\author{
G. Butterweck ${ }^{1, *}$, V. Schmidt ${ }^{2}$, H. Buchröder ${ }^{2}$, R. Hugi ${ }^{1}$, E. Hohmann ${ }^{1}$, E. Foerster ${ }^{2}$ and S. Mayer $^{1}$ \\ ${ }^{1}$ Paul Scherrer Institut, Villigen, Switzerland \\ ${ }^{2}$ Bundesamt für Strahlenschutz, Berlin, Germany \\ *Corresponding author: gernot.butterweck@psi.ch
}

\begin{abstract}
The Bundesamt für Strahlenschutz (Berlin, Germany) and the Paul Scherrer Institute (Villigen, Switzerland) both operate accredited calibration laboratories for radon gas activity concentration. Both the institutions use Lucas Cells as detector in their reference instrumentation due to the low dependence of this detector type on variations in environmental conditions. As a further measure to improve the quality of the reference activity concentration, a spectrometric method of data evaluation has been applied. The electric pulses from the photomultiplier tube coupled to the Lucas Cells are subjected to a pulse height analysis. The stored pulse height spectra are analysed retrospectively to compensate for fluctuations in the electric parameters of the instrumentation during a measurement. The reference instrumentation of both the laboratories is described with the respective spectrum evaluation procedures. The methods of obtaining traceability to the primary calibration laboratories of Germany and Switzerland and data of performance tests are presented.
\end{abstract}

\section{INTRODUCTION}

The accredited calibration laboratories for radon gas concentration measurements at the Bundesamt für Strahlenschutz (BfS) and the Paul Scherrer Institut (PSI) use reference instruments with scintillation cells (Lucas Cells) as detector. The measurement of ${ }^{222} \mathrm{Rn}$ using Lucas Cells was introduced in $1957^{(1)}$. It has since then proved itself in a wide variation of applications as a simple and dependable measuring principle ${ }^{(2-4)}$. Most instruments utilising Lucas Cells separate the pulses originating from electronic noise from alpha particle pulses with a lower-level discriminator (LLD), which is adjusted prior to the measurement in a calibration measurement. An appropriate LLD is coupled to the high voltage applied to the photomultiplier (PM). The combination of both the values is typically chosen to render a low dependence of the instrument efficiency on the high voltage (working point).

The spectrometric approach yields the advantage to determine the LLD retrospectively from the recorded spectra of the measurement. Thus, changes in the PM amplification since the last calibration can be compensated.

At BfS, Lucas Cells are applied as working standard both in grab sampling and in flow-through mode. The radon activity concentration is derived in flowthrough mode from the measured count rate (CR) in a region of interest of the alpha spectrum. Using the grab sampling mode, an ensemble of five cells is flushed with the air of the reference atmosphere. The counting of these five cells starts $3 \mathrm{~h}$ after sampling. The mean value of the CRs of five cells is used to calculate the reference value taking into account the time dependence of the CR. The grab sampling method is used to check the activity concentration values determined with the flow-through method for quality assurance purposes. The grab sampling uses a conventional technique of radiation measurement with a Hamamatsu PM, analogue preamplifier, amplifier, low-level discriminator (Tracerlab $\mathrm{GmbH}$ ), while the CR of flow-through cells is determined using a Hamamatsu PMT in combination with a digital PM base/multichannel combination (ORTEC digibase). The PSI reference instrumentation uses an ensemble of three Lucas Cells in an automated grab sampling mode ${ }^{(5)}$. After a sampling air from the radon chamber, counting starts when radioactive equilibrium between radon and its progeny is reached in the Lucas Cells after $3.5 \mathrm{~h}$. Besides redundancy, the sampling intervals of the individual cells are delayed to improve the time resolution of the measurement. Pylon 300A Lucas Cells in combination with a fixed LLD counter (Pylon AB-5) were used as reference instrumentation in the last two decades at PSI. A replacement of the vintage instruments using a spectrometric approach consisting of Pylon 600A Lucas Cells, Hamamatsu R6231-100 PMs and Canberra Osprey tube base and multichannel combinations was constructed in 2013.

\section{SPECTRUM}

The pulse height spectrum (Figure 1) obtained from the PM shows on the left side a slope resulting from electronic noise, separated by a shallow minimum from the pulses originating from alpha decays of ${ }^{222} \mathrm{Rn},{ }^{218} \mathrm{Po}$ and ${ }^{214} \mathrm{Po}$ on the right side ${ }^{(6)}$. The spectrum depends to some extent on the manufacturer of the Lucas Cell.

BfS decided to precede the introduction of the spectrometric approach with a study on the parameters 


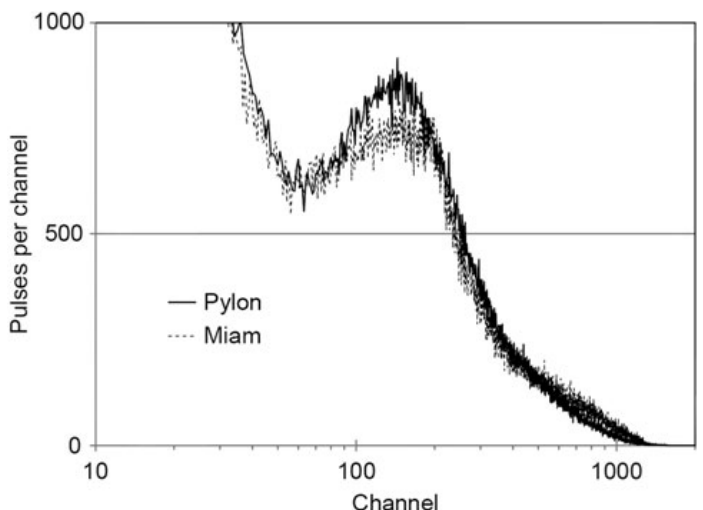

Figure 1. Pulse height spectra obtained with Lucas Cells from different manufacturers.

influencing the alpha spectra of the Lucas cells for spectrometric performance optimisation. With identical cell geometry (cylinder with $5.5 \mathrm{~cm}$ diameter and $12 \mathrm{~cm}$ length), the spectrum shape was strongly influenced by both the method of applying the $\mathrm{ZnS}(\mathrm{Ag})$ layer and the thickness of the layer (Figure 2). The clearer distinction of the counts from alpha particles achieved with liquid painting compared with application via airbrush contradicts published results ${ }^{(7)}$, thus indicating an influence of individual application technique and type of applied $\mathrm{ZnS}(\mathrm{Ag})$ suspension resulting in different layer structures. The thickest $\mathrm{ZnS}(\mathrm{Ag})$ layer tested at BfS up to now was $0.03 \mathrm{~mm}$ on commercially available foils (Eljen Technology). Results ${ }^{(7)}$ published in the literature indicate that sufficient efficiency is reached above 0.027 $\mathrm{mm}$ layer thickness with a maximum at $0.042 \mathrm{~mm}$ thickness. The peak-to-noise ratio improved with increasing layer thickness up to $0.144 \mathrm{~mm}$. The results show the need for further research to optimise the layer properties especially for radon measurements.

\section{SPECTRUM EVALUATION}

Parallel to the optimisation project at BfS, PSI evaluated further steps necessary to render the spectrometric approach into a working reference measuring instrument.

A sum spectrum over the whole measurement duration is used if there is no indication of a change in the spectrum shape during a measurement for the determination of the LLD. Due to rather large statistical fluctuations of the counts per channel, a simple and partly manual approach showed the most robust result in determining the minimum between electronic noise and alpha pulses. The channel of the maximum of the alpha pulses and the channel with the same number of counts in the slope of the electronic noise are marked manually. Ten per cent of the channels in the region between these two markers of counts with the lowest counts per channel are selected. The

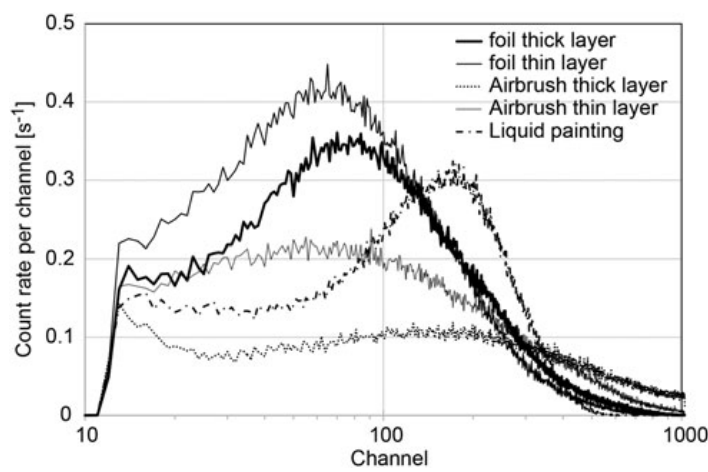

Figure 2. Pulse height spectra obtained with Lucas Cells with different layers of $\mathrm{ZnS}(\mathrm{Ag})$. Foil thick layer $9 \mathrm{mg} \mathrm{cm}^{-2}$; thin layer $3.5 \mathrm{mg} \mathrm{cm}^{-2}$.

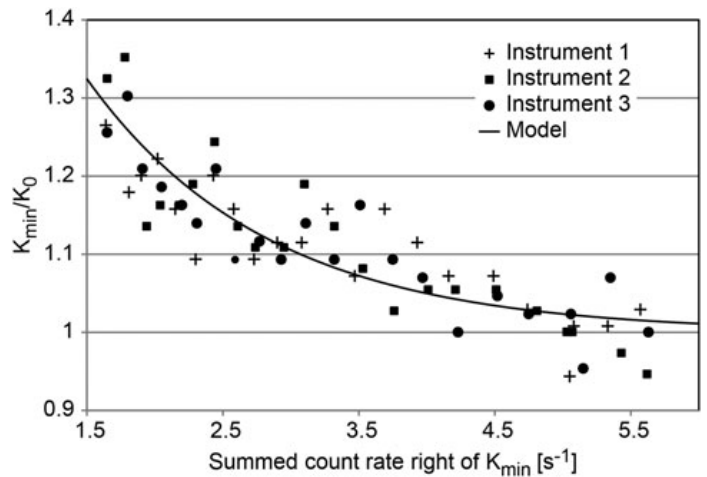

Figure 3. Dependence of the determined minimum channel on the CR.

minimum is set to the arithmetic average of the selected channels. Thus, the determined minimum channel $\left(K_{\min }\right)$ is dependent on the radon concentration. The CR per channel in the alpha pulse region varies with the radon concentration, whereas the slope of the electronic noise is independent of concentration. Therefore, the intersection point between both slopes is a function of the CR (in s ${ }^{-1}$ ) associated with the alpha pulses. A simple exponential model $\left(K_{\min }=K_{0} \times(1+\exp \right.$ $(-0.75 \times C R)))$ is used to take this $\mathrm{CR}$ dependence into account (Figure 3). The minimum channel for high CRs, $K_{0}$, derived with this model is then used as constant LLD for the evaluation of individual spectra of the measurement.

\section{TRACEABILITY}

Reference instruments of accredited laboratories are required to have a calibration traceable to national or international primary standards.

An advantage of Lucas Cells is the possibility to directly calibrate them with radon gas standards by 


\section{G. BUTTERWECK ET AL.}

either injecting radon gas directly into the cell or via a volumetric method, where the radon gas standard is flushed into a larger container and the Lucas Cell is calibrated by sampling from this container ${ }^{(8)}$. PSI uses the volumetric approach. A primary radon gas standard supplied by the Institut de Radiophysique (IRA), Lausanne, Switzerland, is transferred quantitatively into a 201 reference volume calibrated at the Physikalisch-Technische Bundesanstalt (PTB), Braunschweig, Germany. The Lucas Cells are evacuated below an absolute pressure of $2 \mathrm{hPa}$ and then connected to the radon gas atmosphere in the reference volume at normal air pressure. The main contributors to the uncertainty budget of the efficiencies are the uncertainties of the radon gas standard $(50 \%)$, the transfer from the reference volume into the Lucas Cell (25\%), the reference volume $(11 \%)$ and the CR $(13 \%)$. Seven further parameters considered in the uncertainty budget contribute the remaining per cent. Table 1 shows good agreement between the resulting efficiencies for two calibrations using two different radon gas standards with different carrier gases.

\section{PERFORMANCE TESTS}

The spectrometric method was tested in comparison with Lucas Cell reference instruments (Pylon AB5) with a fixed LLD at the working point. Figure 4 compares the measured activity concentrations in the PSI radon chamber averaged over a time interval of 4-31 d. The values are plotted relatively to the plateau average of all six Lucas Cells, separately for each of the three radon concentrations applied. The

Table 1. Summary of results for two different calibrations.

\begin{tabular}{lccc}
\hline Parameter & Instrument 1 & Instrument 2 & Instrument 3 \\
\hline IRA Standard 1310-01.0335, artificial air & & & 126 \\
$\quad K_{0}$ & 86 & 09 & 0.0033 \\
$\quad$ Background $\left(\mathrm{s}^{-1}\right)$ & 0.0055 & 0.0042 & 0.617 \\
$\quad$ Efficiency $\left(\mathrm{s}^{-1} \mathrm{kBq}^{-1} \mathrm{~m}^{3}\right)$ & 0.595 & 0.623 & 119 \\
IRA Standard $1312-01.0336$, nitrogen & 87 & 82 & 0.0032 \\
$K_{0}$ & 0.0048 & 0.0040 & 0.627 \\
${\text { Background }\left(\mathrm{s}^{-1}\right)}_{\text {Efficiency }}^{1}\left(\mathrm{~s}^{-1} \mathrm{kBq}^{-1} \mathrm{~m}^{3}\right)$ & 0.592 & 0.635 & \\
\hline
\end{tabular}

The expanded $(k=2)$ combined uncertainty of all stated efficiencies is $0.0083 \mathrm{~s}^{-1} \mathrm{kBq}^{-1} \mathrm{~m}^{3}$.

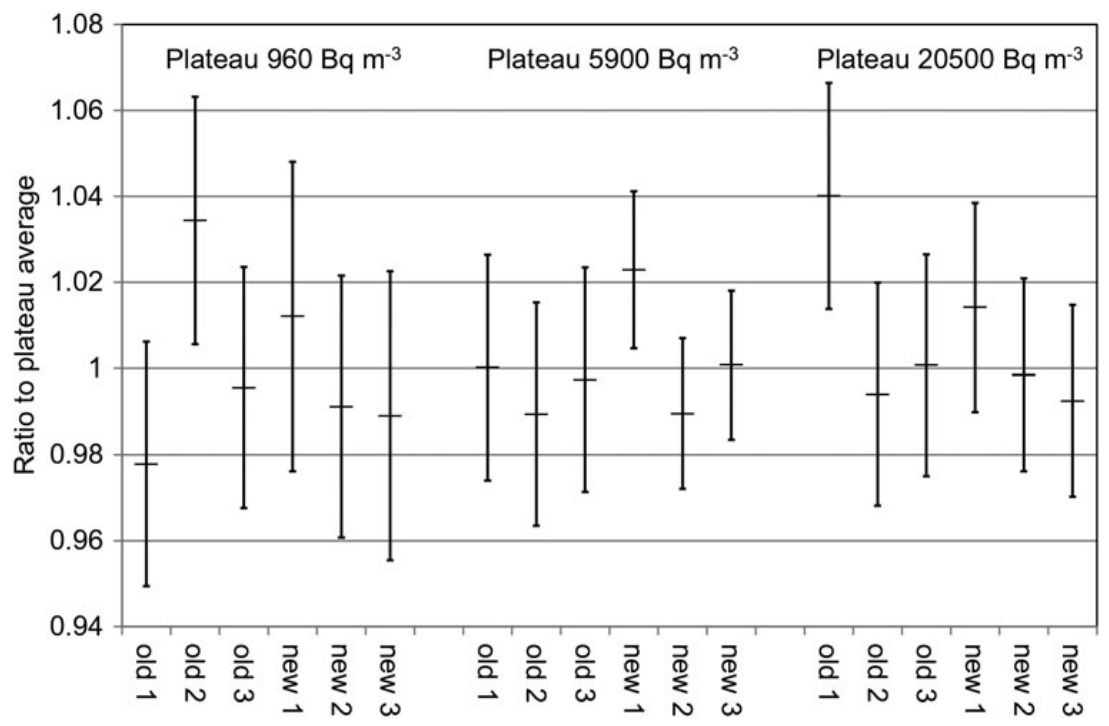

Figure 4. Ratio of radon activity concentrations measured with single Lucas Cells to the average value at the concentration plateau (old 1-3: Lucas Cells with fixed LLD; new 1-3: Lucas Cells with spectrometric evaluation). 
Lucas Cells of the old measuring system show two significant deviations to the average with a maximum value of $4 \%$, whereas the new spectrometric measurement has one significant deviation with a maximum value of $2 \%$.

\section{CONCLUSIONS}

The spectrometric evaluation of pulse height spectra of Lucas Cells yields the opportunity to retrospectively determine a LLD for the separation of noise pulses from pulses originating from radon and radon progeny alpha particles. Thus, the LLD can be monitored and adjusted for each individual measurement, rendering an improved quality of the radon activity concentration reference value in the radon chambers at BfS and PSI. Optimised coating of the $\mathrm{ZnS}(\mathrm{Ag})$ layer can be expected to further improve the performance of the reference measuring systems.

\section{FUNDING}

The presented work was partly funded by the Swiss Federal Office of Public Health.

\section{REFERENCES}

1. Lucas, H. F. Improved low-level alpha-scintillation counter for radon. Rev. Sci. Instrum. 28(9), 680-683 (1957).

2. George, A. C. State-of-the-art instruments for measuring radon/thoron and their progeny in dwellings - a review. Health Phys. 70(4), 451-463 (1996).

3. Vargas, A. and Ortega, X. Influence of environmental changes on continuous radon monitors. Results of a Spanish intercomparision exercise. Radiat. Prot. Dosim. 121(3), 303-309 (2006).

4. Quindos-Poncela, L. S., Fernandez, P. L., Sainz, C., Arteche, J., Arozamena, J. G. and George, A. C. An improved scintillation cell for radon measurements. Nucl. Instr. Meth. Phys. Res. A. 512, 606-609 (2003).

5. Schuler, Ch. Das Referenzlabor für RadongasKonzentrationsmessungen am PSI. PSI-Bericht Nr. 98-08. ISSN 1019-0643. Paul Scherrer Institut (1998).

6. Lenzen, M. and Neugebauer, H. J. A theoretical investigation in the Lucas Cells. Nucl. Instr. Meth. Phys. Res. A. 368, 479-483 (1996).

7. Lee, S. K., Kang, S. Y., Jang, D. Y., Lee, C. H., Kang, S. M., Kang, B. H., Lee, W. G. and Kim, Y. K. Comparison of new simple methods in fabricating $\mathrm{ZnS}(\mathrm{Ag})$ scintillators for detecting alpha particles. Prog. Nucl. Sci. Technol. 1, 194-197 (2011).

8. Gan, T. H., Solomon, S. B. and Peggie, J. R. Standardization of Rn-222 at the Australian radiation laboratory. J. Res. Natl. Inst. Stand. Technol. 95, 171-175 (1990). 